HD-THEP-99-3

TAUP-2548-99

\title{
Odderon and photon exchange in electroproduction of pseudoscalar mesonst
}

\author{
E. R. Berger ${ }^{\mathrm{a}}$, A. Donnachie ${ }^{\mathrm{b}}$ H. G. Dosch ${ }^{\mathrm{a}}$, W. Kilian ${ }^{\mathrm{c}}$, O. Nachtmann ${ }^{\mathrm{a}}$, \\ M. Rueter ${ }^{\mathrm{d}}$ \\ a : Institut für Theoretische Physik der Universität Heidelberg, \\ Philosophenweg 16, D-69120 Heidelberg \\ b : TH Division, CERN; \\ Permanent address: Department of Physics and Astronomy, \\ University of Manchester, Manchester M13 9PL, UK \\ c : Institut für Theoretische Teilchenphysik, \\ Universität Karlsruhe, D-76128 Karlsruhe \\ d : School of Physics and Astronomy, Department of High Energy Physics, \\ Tel Aviv University, 69978 Tel Aviv, Israel
}

\begin{abstract}
We investigate the reaction e $\mathrm{p} \rightarrow$ e PS X where PS denotes a pseudoscalar meson $\pi^{0}, \eta, \eta^{\prime}$, or $\eta_{c}$ and $\mathrm{X}$ either a proton or resonance or continuum states into which the proton can go by diffractive excitation. At high energies photon and odderon exchange contribute to the reaction. The photon exchange contribution is evaluated exactly using data for the total virtual photon-proton absorption cross section. The odderon exchange contribution is calculated in nonperturbative QCD, using functional integral techniques and the model of the stochastic vacuum. For the proton we assume a quark-diquark structure as suggested by the small odderon amplitude in $\mathrm{pp}$ and $\mathrm{p} \overline{\mathrm{p}}$ forward scattering. We show that odderon exchange leads to a much larger inelastic than elastic PS production cross section. Observation of our reaction at HERA would establish the soft odderon as an exchange object on an equal footing with the soft pomeron and would give us valuable insight into both the nucleon structure and the mechanism of high energy diffractive scattering.
\end{abstract}

\footnotetext{
${ }^{1}$ Supported by German Bundesministerium für Bildung und Forschung (BMBF), Contract Nr. 057 HD $91 \mathrm{P}(0)$, by Deutsche Forschungsgemeinschaft under grant no. GKR 216/1-98, by PPARC, by DAAD and MINERVA-Stiftung.

email adresses: e.berger@thphys.uni-heidelberg.de, ad@a3.ph.man.ac.uk, h.g.dosch@thphys.uni-heidelberg.de, kilian@particle.physik.uni-karlsruhe.de, o.nachtmann@thphys.uni-heidelberg.de, rueter@post.tau.ac.il.
} 


\section{Introduction}

The phenomenological (non-perturbative) pomeron has long been established as an effective Regge pole whose exchange governs high-energy diffractive hadron-hadron scattering [1]. The pomeron carries vacuum quantum numbers $C=P=+1$, and there is no a priori reason why a $C=P=-1$ partner, the phenomenological odderon [2], should not exist. If present, the exchange of an odderon Regge pole would produce a difference between pp and $\bar{p}$ scattering at high energies and at small momentum transfer. A particularly sensitive test is provided by measurements of the forward real part of the pp and $\mathrm{p} \overline{\mathrm{p}}$ scattering amplitudes, and they are consistent with the absence of odderon exchange [3]. There are two possible explanations for the apparant absence of the odderon. One is that the nonperturbative odderon really does not exist. This seems implausible as QCD-based models of the phenomenological pomeron can easily be extended to describe a phenomenological odderon. Further a perturbative "odderon", namely three-gluon exchanget is believed to dominate large-angle pp and $\mathrm{p} \overline{\mathrm{p}}$ scattering. One of the most compelling arguments for a $C=-1$ exchange in high energy scattering is provided by the pp and $p \bar{p}$ differential cross sections at the ISR where a deep dip in the former process is transformed into a shoulder in the latter. The second possibility is that the phenomenological odderon does exist, but that its coupling to the nucleon in elastic scattering at small $t$ is extremely small. That is the view we take here.

One successful approach to high-energy diffractive scattering is based on functional integral techniques [9, 10] and the use of the model of the stochastic vacuum (MSV) [11] to evaluate the correlation functions of the Wegner-Wilson loops which occur in the formalism when applied to hadron-hadron scattering [12, 13]. This gives a remarkably good description of many different processes involving the exchange of vacuum quantum numbers [13, 14, 15, 16, [17. This model can easily be extended to the exchange of a $C=P=-1$ object, and it has been shown that the clustering of two quarks to form a diquark in a nucleon leads to a drastic reduction in the odderon- $N-N$ coupling [18]. The general result is rather model independent. It relies on the fact that the quark-diquark density in a nucleon is nearly symmetric under a parity transformation (if the diquark is sufficiently small) whereas the odderon coupling changes sign. Therefore there is a cancellation when the nucleon wave function is integrated over all angles. Specifically, in the MSV it has been shown that the formation of a diquark with a radius $r_{D}<0.3 \mathrm{fm}$ yields sufficient suppression of the odderon coupling to the proton in order to be in agreement with the upper limit allowed by the measurements of the forward real part of the pp and p $\bar{p}$ scattering amplitudes.

It should be stressed that this argument is only valid if the odderon is treated as a simple Regge pole near $J=1$. If a more complicated situation is permitted, as in [19], then

\footnotetext{
${ }^{2} \mathrm{~A}$ lot of theoretical work has been devoted to calculating perturbative QCD (pQCD) corrections to this type of odderon [4, 6, 6, 7]. The result seems to be that such corrections have a small effect, for

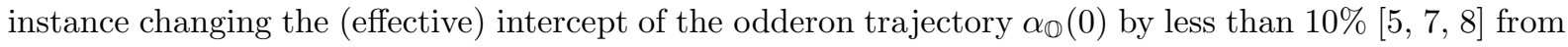
the value of 1 , which is the result for the lowest order three gluon exchange.
} 
the phenomenology is very different and the suppression of odderon exchange in pp and $\mathrm{p} \overline{\mathrm{p}}$ scattering at high energies is much less marked. However the data do not require the additional complexity of double and triple poles, so until demonstrated explicitly otherwise we prefer to stay with the simplest model which agrees with data and which allows essentially parameter-free predictions.

It is clearly advantageous if one can find high-energy reactions which permit odderon exchange but exclude pomeron exchange. Exclusive neutral pseudoscalar meson production in ep scattering at high energies is one such, providing a direct probe for odderon exchange [20, 21]. Using a simple ansatz for the odderon, this cross section has been calculated [22. Because of the suppression of the odderon-p-p coupling, the cross section due to photon-photon fusion is comparable to that expected at best from odderon exchange. Nonetheless there are kinematical distributions which could provide promising signals for the odderon.

The suppression of the odderon coupling due to diquark formation does not hold if the nucleon is transformed diffractively into an excited negative parity state. In this case, even for a pointlike diquark the odderon couples to the nucleon without any restriction 23] giving promise of a significantly higher cross section for odderon exchange in, for example, ep $\rightarrow \mathrm{e} \pi^{0} \mathrm{X}$ (where $\mathrm{X}$ stands for diffractively excited proton states) compared to $\mathrm{ep} \rightarrow \mathrm{e} \pi^{0} \mathrm{p}$. In fact this is much more representative of the real experimental situation, as it is often not possible to say whether the proton has recoiled quasi-elastically or has been transformed into an excited state within some experimentally defined mass range. At HERA the upper limit for the recoil mass $M_{X}$ is typically $\sim 2 \mathrm{GeV}$. Accordingly we calculate the contribution from odderon exchange to pseudoscalar meson production with nucleon fragmentation in ep interactions at high energies, Fig.1(a). To complement this we also calculate the contribution to the same process from photon exchange, Fig.1(b). This latter calculation is exact, limited only by the errors on the ep total cross section for the $\left(Q^{2}, M_{X}\right)$ range of relevance. When combined with the previous calculation [22] of photon exchange for the quasi-elastic process this provides an absolute prediction for the cross section from the electromagnetic process alone. Any measurement deviating significantly from this would be strong evidence for the odderon. Thus we are considering electroproduction of a pseudoscalar meson $\mathrm{PS}=\pi^{0}, \eta, \eta^{\prime}, \eta_{c}$ with nucleon break-up:

$$
\mathrm{e}^{ \pm}\left(p_{1}\right)+\mathrm{p}(p) \rightarrow \mathrm{e}^{ \pm}\left(p_{1}^{\prime}\right)+\mathrm{PS}(k)+\mathrm{X}\left(p_{X}\right) .
$$

We define $q_{1}=p_{1}-p_{1}^{\prime}, q_{2}=q_{1}-k=p_{X}-p, s=\left(p_{1}+p\right)^{2}, W=s_{2}=\left(q_{1}+p\right)^{2}$, $t_{1}=q_{1}^{2}=-Q^{2}$ and $t_{2}=q_{2}^{2}$. Here we treat the very small $Q^{2}$ range. In the H1 experiment at HERA the kinematical cuts for this so called photoproduction region [24] are

$$
\begin{aligned}
& y_{\min }=0.3 \leq y \leq 0.7=y_{\max } \\
& 0<Q^{2}<0.01 \mathrm{GeV}^{2}
\end{aligned}
$$

where, in the proton rest frame $y=\left(p q_{1}\right) /\left(p p_{1}\right)$ is the fractional energy loss of the incoming lepton. Due to the cuts (2) the photons emitted by the $\mathrm{e}^{ \pm}$are always nearly on shell, and 


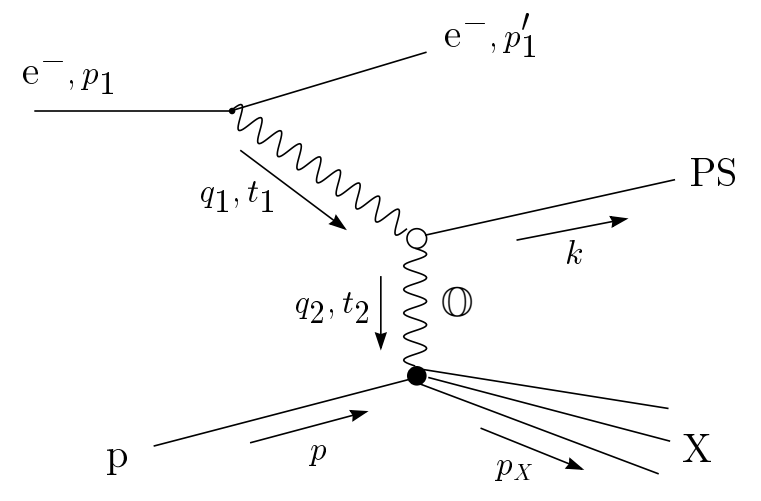

(a)

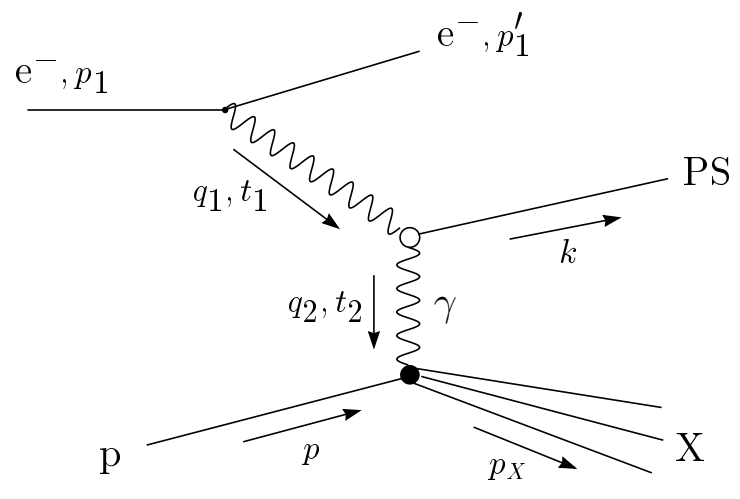

(b)

Figure 1: Feynman diagrams for pseudoscalar meson production in ep scattering at high energies with odderon (a) and photon (b) exchange.

the equivalent photon approximation (EPA) [25] is applicable. The total electroproduction cross section for producing a PS in terms of the EPA is given by

$$
\begin{gathered}
\sigma=\int_{y_{\min }}^{y_{\max }} \frac{d y}{y} n(y) \sigma_{\gamma \mathrm{p}}\left(s_{2}\right) \quad \text { with } \quad s_{2}=y s+(1-y) m_{\mathrm{p}}^{2}, \\
n(y)=\frac{\alpha}{\pi}\left\{\left(1-y+\frac{y^{2}}{2}\right) \ln \left(\frac{|t|_{u}}{|t|_{l}}\right)-\frac{m_{e}^{2} y^{2}}{|t|_{l}}\left(1-\frac{|t|_{l}}{|t|_{u}}\right)-\right. \\
\left.\left(1-\frac{y}{2}\right)^{2} \ln \left(\frac{|t|_{u} / E^{2}+y^{2}}{|t|_{l} / E^{2}+y^{2}}\right)\right\},
\end{gathered}
$$

where $m_{\mathrm{p}}$ is the nucleon mass and $\sigma_{\gamma \mathrm{p}}$ is the total photoproduction cross section for the reaction. $n(y)$ is the equivalent photon number [25] for a given energy fraction of the incoming $\mathrm{e}^{ \pm}$transfered to the $\gamma \mathrm{p}$ subsystem. In the proton rest frame $E=p p_{1} / m_{\mathrm{p}}$ is the energy of the incoming $e^{ \pm}$. The upper limit $|t|_{u}$ is given by experiment (2) $),|t|_{u}=0.01$ $\mathrm{GeV}^{2}$, and $|t|_{l}=m_{e}^{2} y^{2} /(1-y)$.

\section{The odderon contribution}

For convenience we discuss the odderon exchange contribution to the reaction $\gamma \mathrm{p} \rightarrow \pi^{0} \mathrm{X}$. The conversion to an incident electron via the EPA is in principle straightforward, as is 
the replacement of the $\pi^{0}$ by one of the other pseudoscalars.

There are two apparently extreme approaches to this calculation. One is to assume that the system $\mathrm{X}$ is dominated by a small number of resonances, which for the odderon should be a good approximation. The second is to ignore any possible structure in the final system $\mathrm{X}$ and represent it simply by a free quark-diquark pair. Both calculations are performed and give similar results.

We discuss first resonance production. The break-up of the nucleon by odderon exchange leads naturally to negative parity final states. The lowest lying nucleonic isoscalar states with negative parity are the $N(1520): J^{P}=\frac{3}{2}^{-}$and the $N(1535): J^{P}=\frac{1}{2}^{-}$. These are both compatible with the diquark picture. For dynamical reasons a scalar diquark is favoured over a vector one [26]. The P-wave excitation of a quark and a scalar diquark gives degenerate nucleon resonances with quantum numbers $J^{P}=\frac{3}{2}^{-}, \frac{1}{2}^{-}$which can readily be identified with these two observed states.

It is easy to see that considering unpolarised cross sections, summed over both resonances the quark spin degree of freedom becomes irrelevant and the calculation reduces to one where a spinless state is exited to a $2 \mathrm{P}$ resonance.

The scattering amplitude $T\left(s_{2}, t_{2}\right)$, calculated in the $\gamma \mathrm{p}$ c.m. system, can be expressed through a profile function $J(\boldsymbol{b})$ :

$$
T\left(s_{2}, t_{2}\right)=2 i s_{2} \int d^{2} b e^{i \boldsymbol{q}_{\mathbf{2}_{T}} \boldsymbol{b}} J(\boldsymbol{b})
$$

The profile function is expressed in the model [13] as an overlap of a dipole-dipole scattering amplitude $\tilde{J}\left(\boldsymbol{b}, \boldsymbol{r}_{1}, z_{1}, \boldsymbol{r}_{2}, z_{2}\right)$ where $\boldsymbol{b}$ is the impact parameter of two lightlike dipole trajectories with (transverse) size $\boldsymbol{r}_{1}$ and $\boldsymbol{r}_{2}$ respectively. The quantities $z_{1}, z_{2}$ are the longitudinal momentum fractions of the quarks in the dipoles.

It is of course necessary to take a profile function corresponding to the exchange of a $C=P=-1$ object [18. Note that the model contains only the kinematical $s_{2}$-dependence shown in (14) which leads to an energy independent cross section. The parameters of the model[ are the same as those used in 23] which were fixed at an energy of $\sqrt{s_{2}}=W=20$ $\mathrm{GeV}$. We return to the question of energy dependence at the end of this section.

Hadronic amplitudes for production of hadronic resonance states are obtained by smearing the dipole extensions $\boldsymbol{r}_{i}$ with the respective transition densities:

$$
\begin{aligned}
J(\boldsymbol{b})_{\lambda, \lambda_{\gamma}}= & \int \frac{d^{2} r_{1}}{4 \pi} d z_{1} \int \frac{d^{2} r_{2}}{4 \pi} \\
& \sum_{f, h_{1}, h_{2}} \Psi_{f h_{1} h_{2}}^{* \pi^{0}}\left(\boldsymbol{r}_{1}, z_{1}\right) \Psi_{\lambda_{\gamma}, f h_{1} h_{2}}^{\gamma}\left(\boldsymbol{r}_{1}, z_{1}\right) \Psi_{\lambda}^{* 2 \mathrm{P}}\left(\boldsymbol{r}_{2}\right) \Psi^{\mathrm{p}}\left(\boldsymbol{r}_{2}\right) \tilde{J}\left(\boldsymbol{b}, \boldsymbol{r}_{1}, z_{1}, \boldsymbol{r}_{2}\right) .
\end{aligned}
$$

${ }^{3}$ The parameters of the pion-photon overlap can be found in [23]. The MSV parameters are: $a=0.31$ fm, $\left\langle g^{2} F F\right\rangle=3.0 \mathrm{GeV}^{4}, S_{\mathrm{p}}=0.85 \mathrm{fm}$ as determined in [27]. 
Here $\lambda, \lambda_{\gamma}$ stands for the helicities of the photon and of the the orbital helicity of the resonance, respectively. In agreement with the other applications of the model we use for the quark-diquark wave function of the proton a Gaussian with an extension parameter $S_{\mathrm{p}}$ adjusted to $p p$ scattering and neglect the dependence on the longitudinal momentum fraction $z_{2}$ in the purely hadronic overlap. Thus we take for the proton wave function

$$
\Psi^{\mathrm{p}}\left(r_{2}\right)=\frac{\sqrt{2} e^{-r_{2}^{2} / 4 S_{\mathrm{p}}^{2}}}{S_{\mathrm{p}}} .
$$

As the orbital wave function for the low lying degenerate excited states we choose an ansatz analogous to the proton-wave function but in a P-state:

$$
\begin{aligned}
& \Psi_{\lambda}^{2 \mathrm{P}}\left(\boldsymbol{r}_{2}\right)=\Psi^{2 \mathrm{P}}\left(r_{2}\right) e^{i \lambda \theta_{2}}, \\
& \Psi^{2 \mathrm{P}}\left(r_{2}\right)=\frac{r_{2} e^{-r_{2}^{2} / 4 S_{\mathrm{p}}^{2}}}{S_{\mathrm{p}}^{2}}
\end{aligned}
$$

with the same extension parameter $S_{\mathrm{p}}$ as for the proton. An analogous strategy has been applied successfully for the excited $\rho$ states [16]. In (田) we use $\boldsymbol{q}_{\mathbf{2}_{T}}$ as x-axis for the transverse vectors $\boldsymbol{r}_{1}, \boldsymbol{r}_{2}$ and $\boldsymbol{b}$, so $\theta_{2}$ is the angle of $\boldsymbol{r}_{2}$ in planar coordinates. The orbital helicity $\lambda$ of the $2 \mathrm{P}$ state can take the values $0, \pm 1$, but the orbital helicity $\lambda=0$ cannot be excited in our model.

The photon-pion overlap is taken from [23]:

$$
\begin{aligned}
& \sum_{f, h_{1}, h_{2}} \Psi_{f h_{1} h_{2}}^{* \pi^{0}}\left(\boldsymbol{r}_{1}, z_{1}\right) \Psi_{\lambda_{\gamma}, f h_{1} h_{2}}^{\gamma}\left(\boldsymbol{r}_{1}, z_{1}\right)= \\
& i \frac{e}{\sqrt{2}} f_{\pi} e^{-\omega^{2} r_{1}^{2} / 2} e^{i \lambda_{\gamma} \theta_{2}} z_{1}\left(1-z_{1}\right) f\left(z_{1}\right)\left(m_{q} \mathrm{~K}_{1}\left(m_{q} r_{1}\right)+r_{1} \omega^{2} \mathrm{~K}_{0}\left(m_{q} r_{1}\right)\right)
\end{aligned}
$$

Here $m_{q}$ is the quark mass, $m_{q}=0.22 \mathrm{GeV}$. For a justification of such a simple ansatz see [15]. It should be noted that the $\gamma \gamma$ decay width of the $\pi^{0}$ comes out correctly with this overlap. The function $f\left(z_{1}\right)$ is given by [28]:

$$
f\left(z_{1}\right)=\mathcal{N} \sqrt{z_{1}\left(1-z_{1}\right)} \exp \left(-\frac{M_{\pi}^{2}\left(z_{1}-1 / 2\right)^{2}}{2 \omega^{2}}\right)
$$

and $\omega$ and $\mathcal{N}$ are fixed by normalization.

To take advantage of global azimuthal invariance it is useful to choose as new integration variables the relative angles between $\boldsymbol{b}$ and $\boldsymbol{r}_{1(2)} \theta_{1(2)}^{\prime}=\theta_{1(2)}-\theta_{b}$. Then with this choice of coordinates $\tilde{J}$ in (5) becomes independent of $\theta_{b}$ and inserting (6), (7) and (8) into the scattering amplitude (4) we get

$$
\begin{aligned}
T_{\lambda, \lambda_{\gamma}}= & 2 i s_{2} \int b d b d \theta_{b} e^{i \sqrt{-t_{2}} b \cos \left(\theta_{b}\right)} \int \frac{d^{2} r_{1}}{4 \pi} d z_{1} \int \frac{d^{2} r_{2}}{4 \pi} \\
& \times\left[\sum_{f, h_{1}, h_{2}} \Psi_{f h_{1} h_{2}}^{* \pi^{0}}\left(r_{1}, z_{1}\right) \Psi_{\lambda_{\gamma}, f h_{1} h_{2}}^{\gamma}\left(r_{1}, z_{1}\right)\right]_{\theta_{1}=0} \Psi^{* 2 P}\left(r_{2}\right) \Psi^{\mathrm{P}}\left(r_{2}\right) \\
& \times e^{i \lambda_{\gamma}\left(\theta_{1}^{\prime}+\theta_{b}\right)} e^{i \lambda\left(\theta_{2}^{\prime}+\theta_{b}\right)} \tilde{J}\left(\boldsymbol{b}, \boldsymbol{r}_{1}, z_{1}, \boldsymbol{r}_{2}\right)
\end{aligned}
$$


where the wavefunctions now only depend on $r_{1(2)}$. Using the relation

$$
\int_{0}^{2 \pi} d \theta_{b} e^{i \sqrt{-t_{2}} b \cos \left(\theta_{b}\right)} e^{i n \theta_{b}}=(i)^{n} 2 \pi J_{n}\left(\sqrt{-t_{2}} b\right)
$$

we can now perform the $\theta_{b}$ integration and obtain finally for the scattering amplitude

$$
\begin{aligned}
T_{\lambda, \lambda_{\gamma}}= & 2 i s_{2} \int b d b \int \frac{d^{2} r_{1}}{4 \pi} d z_{1} \int \frac{d^{2} r_{2}}{4 \pi} \\
\times & {\left[\sum_{f, h_{1}, h_{2}} \Psi_{f h_{1} h_{2}}^{* \pi^{0}}\left(r_{1}, z_{1}\right) \Psi_{\lambda_{\gamma}, f h_{1} h_{2}}^{\gamma}\left(r_{1}, z_{1}\right)\right]_{\theta_{1}=0} \Psi^{* 2 \mathrm{P}}\left(r_{2}\right) \Psi^{\mathrm{P}}\left(r_{2}\right) } \\
& \times e^{i\left(\lambda_{\gamma} \theta_{1}^{\prime}+\lambda \theta_{2}^{\prime}\right)}(-i)^{\left(\lambda+\lambda_{\gamma}\right)} 2 \pi J_{\left(\lambda+\lambda_{\gamma}\right)}\left(\sqrt{-t_{2}} b\right) \tilde{J}\left(\boldsymbol{b}, \boldsymbol{r}_{1}, z_{1}, \boldsymbol{r}_{2}\right) .
\end{aligned}
$$

The resulting differential cross section is:

$$
\frac{d \sigma_{\gamma \mathrm{p}}^{\mathbb{D}}}{d t_{2}}=\frac{1}{16 \pi s_{2}^{2}} \frac{1}{2} \sum_{\lambda} \sum_{\lambda_{\gamma}}\left|T_{\lambda, \lambda_{\gamma}}\right|^{2} .
$$

One of the features of the result (13) is, that the differential cross section in the forward direction $\left(t_{2}=0\right)$ does not vanish due to the appearence of the Bessel function $J_{0}$ in (12) for $\lambda+\lambda_{\gamma}=0$. The differential cross section (13) for photoproduction is displayed in Fig. 2. The integrated cross section is:

$$
\sigma_{\gamma \mathrm{p}}^{\mathbb{Q}}\left(\gamma \mathrm{p} \rightarrow \pi^{0}\{2 \mathrm{P}\}\right)=294 \mathrm{nb}
$$

As this photoproduction cross section is constant, i.e. independent of $s_{2}$, the EPA conversion to electroproduction can be achieved by simply multiplying it with a constant $c_{\mathrm{EPA}}=0.0136$ corresponding to the $y$ integration in $(3)$. This gives

$$
\sigma^{\mathbb{O}}\left(\mathrm{ep} \rightarrow \mathrm{e} \pi^{0}\{2 \mathrm{P}\}\right)=4.01 \mathrm{nb}
$$

The other possibility, namely treating the final state $X$ in (5) as a free quark-diquark pair, is achieved by approximating the final state by a plane wave. This is effectively invoking quark-hadron duality, and should be a reasonable approximation if we are not interested in the local form of the mass spectrum of $X$ but only in the integral over the full mass spectrum. In this case the integrated cross section was found to be [23]

$$
\sigma_{\gamma \mathrm{p}}^{\mathbb{O}}\left(\gamma \mathrm{p} \rightarrow \pi^{0} \mathrm{X}\right)=341 \mathrm{nb}
$$

consistent with (14).

A good check for the use of quark-hadron duality in diffractive processes is provided by the application of a similar method to the reaction $\gamma \mathrm{p} \rightarrow \rho \mathrm{X}$ and its comparison with elastic $\rho$ photoproduction. One obtains in the model [16]:

$$
\sigma(\gamma \mathrm{p} \rightarrow \rho \mathrm{p})=7.9 \mu \mathrm{b}
$$




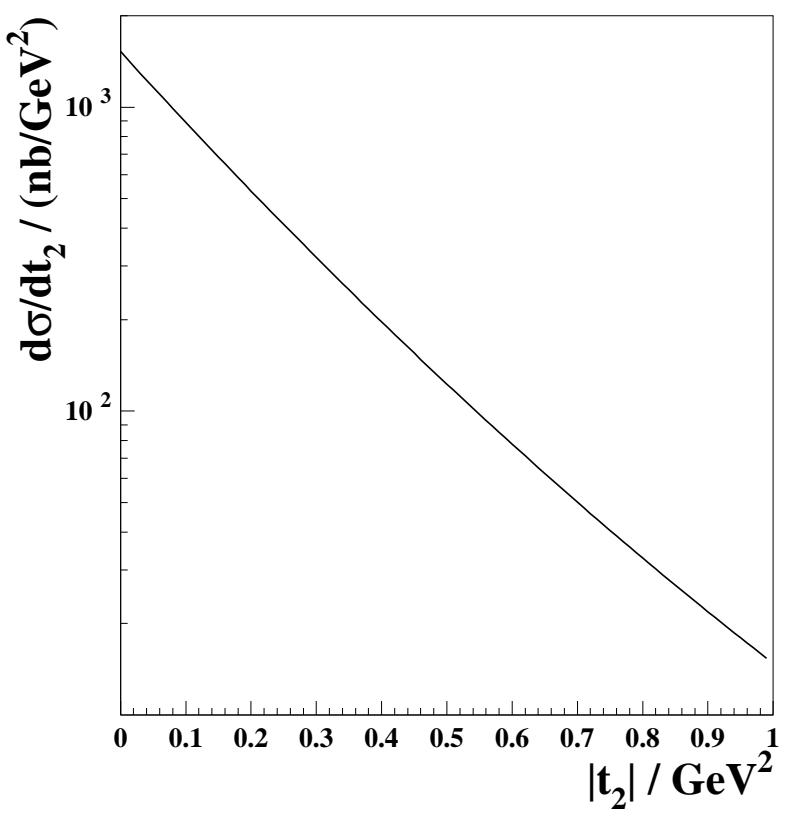

Figure 2: The differential cross section $d \sigma_{\gamma \mathrm{p}}^{\mathbb{Q}} / d t_{2}$ of the process $\gamma \mathrm{p} \rightarrow \pi^{0}\{2 \mathrm{P}\}$ as a function of $t_{2}$. (A fit to this curve is: $d \sigma_{\gamma \mathrm{p}}^{\mathbb{O}} / d t_{2}=a \exp \left(-b t_{2}-c t_{2}^{2}\right)$ where $a=1523 \mathrm{nb}, \quad b=$ $\left.5.44 \mathrm{GeV}^{-2}, c=-0.80 \mathrm{GeV}^{-4}\right)$

in good agreement with the pomeron contribution 29] at $W=20 \mathrm{GeV}$ to the total $\rho$ photoproduction cross section [30]. For the ratio one obtainst

$$
\frac{\sigma(\gamma \mathrm{p} \rightarrow \rho \mathrm{X})}{\sigma(\gamma \mathrm{p} \rightarrow \rho \mathrm{p})} \approx 1.5
$$

in agreement with the data at HERA energies 31.

It should be noted that the results for diffractive dissociation depend much more on the choice of the wave functions than for elastic processes. In the latter the overlap becomes essentially the density and is constrained by normalization. The photon-pion overlap has been tested to some extent by the pion radiative decay, but there is no such test for the proton-2P overlap. The odderon contribution is also much more sensitive to the parameters of the MSV than pomeron exchange. We have also applied the matrix cumulant expansion technique [17], and with the approximations as done there we found results differing from the above ones at most by $50 \%$. Taking all these uncertainties into account we estimate the uncertainty in the cross section in this model calculation to be at least a factor 2 at $W=20 \mathrm{GeV}$.

Finally we return to the question of energy-dependence. In hadron hadron scattering the increase of the cross sections together with the shrinking of the diffraction peak can be well

\footnotetext{
${ }^{4}$ We thank S. Weinstock for communicating this result prior to publication.
} 
reproduced in this model by scaling the hadronic radii by $\left(W^{2} / 400 \mathrm{GeV}^{2}\right)^{0.08 / 3}$ [13, 17]. Assuming that the same radial scaling reproduces the energy dependence of the odderon contributions we find that the integrated cross section scales like $\left(W^{2} / 400 \mathrm{GeV}^{2}\right)^{0.15}$, leading to an enhancement of ca. 1.8 for HERA energies as compared to $\sqrt{W}=20 \mathrm{GeV}$.

\section{The electromagnetic cross section}

In this section we consider PS production mediated by photon rather than odderon exchange (Fig. 1b). Again the proton, now hit by the photon, is allowed to go into some hadronic final state.

The coupling of a pseudoscalar meson PS to two photons is fixed as described in [22]. The Lorenz structure is that of the triangle anomaly where the strength of the coupling, parametised by a constant $u_{P S}$, can be extracted from the partial decay width of the PS decaying into two photons. The scattering amplitude in leading order perturbation theory of the electroweak interaction is

$$
\begin{aligned}
S_{f i}= & i(2 \pi)^{4} \delta^{4}\left(p+p_{1}-p_{1}^{\prime}-k-p_{X}\right) T_{f i}, \\
T_{f i}= & -e \bar{u}\left(p_{1}^{\prime}\right) \gamma^{\mu} u\left(p_{1}\right) \frac{1}{t_{1}} u_{P S} \epsilon_{\mu \nu \rho \sigma} q_{1}^{\rho} q_{2}^{\sigma} T\left(t_{1}, t_{2}\right) \frac{1}{t_{2}} \\
& \times\left\langle X\left(p_{X}\right)\left|e J^{\nu}(0)\right| P(p)\right\rangle .
\end{aligned}
$$

Here $J^{\nu}$ is the hadronic part of the electromagnetic current and the form factor $T\left(t_{1}, t_{2}\right)$ is given in terms of a vector meson dominance ansatz (cf. [22]).

We are not interested in properties due to polarisation of the initial and final state particles and so average over the initial particle polarisations and sum over the final ones. In addition we are also not interested in momentum distributions of the outgoing hadrons except, clearly, for the PS. For this reason we perform the integrations over their momenta.

Summing over all kinematically accessible states $\mathrm{X}$ the differential cross section can be written as

$$
\begin{aligned}
& d^{6} \sigma^{\gamma}=\frac{1}{2 w\left(s, m^{2}, m_{e}^{2}\right)} \frac{d^{3} p_{1}^{\prime}}{(2 \pi)^{3} 2 p_{1}^{\prime 0}} \frac{d^{3} k}{(2 \pi)^{3} 2 k^{0}} \rho^{\mu \nu} P_{\mu \nu}, \\
& \rho^{\mu \nu}:=\frac{e^{2}}{t_{1}^{2}}\left\{\left(g^{\mu \nu}-\frac{q_{1}^{\mu} q_{1}^{\nu}}{q_{1}^{2}}\right)+\frac{\left(2 p_{1}-q_{1}\right)^{\mu}\left(2 p_{1}-q_{1}\right)^{\nu}}{q_{1}^{2}}\right\}, \\
& P_{\mu \nu}:=\frac{e^{2}}{t_{2}^{2}} u_{P S}^{2} T^{2}\left(t_{1}, t_{2}\right) \epsilon_{\mu \omega \alpha \beta} q_{1}^{\alpha} q_{2}^{\beta} \epsilon_{\nu \rho \gamma \delta} q_{1}^{\gamma} q_{2}^{\delta}(2 \pi)\left(2 m_{\mathrm{p}}\right) W^{\rho \omega}, \\
& w(x, y, z):=\left(x-(\sqrt{y}+\sqrt{z})^{2}\right)^{\frac{1}{2}}\left(x-(\sqrt{y}-\sqrt{z})^{2}\right)^{\frac{1}{2}} .
\end{aligned}
$$

Here $W_{\rho \omega}$ is the usual hadron tensor as defined for example in chapter 18 of [32] involving the two invariant functions $W_{1}$ and $W_{2}$. If the sum over all kinematically accessible

\footnotetext{
${ }^{5}$ Throughout this Section all notations are as in 32.
} 
states is restricted to some subset invariant under Lorentz and parity transformations the two invariant functions $W_{1,2}$ which appear in $W_{\rho \omega}$ change, whereas the tensor structures, multiplied by $W_{1,2}$, do not. The photoproduction cross section of (3) for photon exchange $\sigma_{\gamma \mathrm{p}}\left(s_{2}\right)$ is given by

$$
\begin{gathered}
\sigma_{\gamma \mathrm{p}}^{\gamma}\left(s_{2}\right)=\int \frac{d^{3} k}{2 k^{0}(2 \pi)^{3}} \frac{1}{2 w\left(s_{2}, m^{2}, t_{1}\right)} \frac{1}{2}\left(-g^{\mu \nu}\right) \\
\left(u_{P S}^{2} T^{2} e^{2} \epsilon_{\mu \omega \alpha \beta} q_{1}^{\alpha} q_{2}^{\beta} \epsilon_{\nu \rho \gamma \delta} q_{1}^{\gamma} q_{2}^{\delta}\right) \frac{1}{t_{2}^{2}} \\
(2 \pi)\left(2 m_{\mathrm{p}}\right) W^{\rho \omega} .
\end{gathered}
$$

We now evaluate (21) using the data on total $\gamma^{*}$ p cross section collected in [33 where the $\gamma^{*}$ p c.m. energy is below $2.0 \mathrm{GeV}$ and $0 \leq\left|t_{2}\right| \leq 6.0 \mathrm{GeV}^{2}$. As a check on the procedure we compare the result for the $\Delta(1232)$ resonance region with an explicit calculation of $\Delta$ electroproduction.

\subsection{The resonance region}

The total $\gamma^{*}$ p absorption cross section $\Sigma=\sigma_{T}+\epsilon \sigma_{L}$ in the region of the main nucleon resonances has been determined in inelastic electron proton scattering experiments. With the kinematical definitions of [34] the relation of $\sigma_{T}$ and $\sigma_{L}$ to $W_{1,2}$ is:

$$
\begin{aligned}
& W_{1}\left(M_{X}^{2}, q_{2}^{2}\right)=\frac{1}{4 \pi^{2} \alpha} \frac{M_{X}^{2}-m_{\mathrm{p}}^{2}}{2 m_{\mathrm{p}}} \sigma_{T}\left(M_{X}^{2}, q_{2}^{2}\right), \\
& W_{2}\left(M_{X}^{2}, q_{2}^{2}\right)=\frac{1}{4 \pi^{2} \alpha} \frac{M_{X}^{2}-m_{\mathrm{p}}^{2}}{2 m_{\mathrm{p}}}\left(1-\nu^{2} / q_{2}^{2}\right)^{-1}\left[\sigma_{T}\left(M_{X}^{2}, q_{2}^{2}\right)+\sigma_{L}\left(M_{X}^{2}, q_{2}^{2}\right)\right], \\
& \nu=\frac{1}{2 m_{\mathrm{p}}}\left(M_{X}^{2}-m_{\mathrm{p}}^{2}-q_{2}^{2}\right) .
\end{aligned}
$$

Here $\alpha$ is the fine structure constant. For very small photon virtualities there are photoproduction data in addition. In [33] all these data have been combined to extract the following parametrisation of $\Sigma$ as a function of the photon virtuality $q_{2}^{2}$ and the invariant mass squared of the final state $M_{X}^{2}$

$$
\begin{aligned}
& \ln \left(\frac{\Sigma}{G_{D}^{2}}\right)=a\left(M_{X}^{2}\right)+b\left(M_{X}^{2}\right) \ln \left(\frac{\left|\boldsymbol{q}_{2}\right|}{\left|\boldsymbol{q}_{2}\right|_{0}}\right)+c\left(M_{X}^{2}\right)\left|\ln \left(\frac{\left|\boldsymbol{q}_{\mathbf{2}}\right|}{\left|\boldsymbol{q}_{2}\right|_{0}}\right)\right|^{d\left(M_{X}^{2}\right)}, \\
& G_{D}\left(q_{2}^{2}\right)=\frac{1}{\left(1-q_{2}^{2} / 0.71 \mathrm{GeV}^{2}\right)^{2}} .
\end{aligned}
$$

$G_{D}$ is the dipole form factor, $\left|\boldsymbol{q}_{2}\right|\left(\left|\boldsymbol{q}_{2}\right|_{0}\right)$ is the absolute value of the photon momentum in the proton rest frame for photon virtuality $q_{2}^{2}\left(q_{2}^{2}=0\right)$. The fit is restricted to the range $1.11 \leq M_{X} \leq 1.99 \mathrm{GeV}$. The functions $a, b, c$ are given in [33 only at discrete points?.

\footnotetext{
${ }^{6}$ In the range $1.11 \leq M_{X} \leq 1.755$ in steps of $\Delta M_{X}=0.015 \mathrm{GeV}$ and in the range $1.755 \leq M_{X} \leq 1.990$ in steps of $\Delta M_{X}=0.02 \mathrm{GeV}$.
} 
The function $d$ is taken to be a constant, $d=3.0$. To convert the fit to a continuous function in $M_{X}^{2}$ and $q_{2}^{2}$ we represent $\Sigma$ as a histogram. Fig. 3 shows as an example $\Sigma$ for a fixed photon virtuality of $q_{2}^{2}=-0.1 \mathrm{GeV}^{2}$.

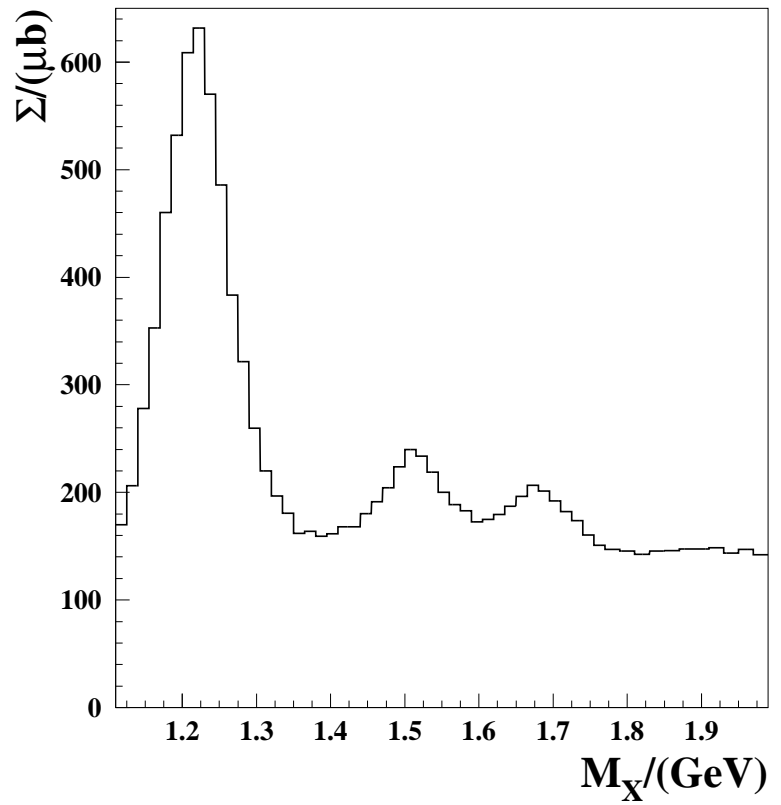

Figure 3: The $\gamma^{*}$ p absorption cross section $\Sigma$ for $q_{2}^{2}=-0.1 \mathrm{GeV}^{2}$ as a histogram using the parametisation (23)

It was shown in [33] that the total virtual photon-proton cross section for longitudinal photons $\sigma_{L}$ is small in the resonance region, so we neglect it in our discussion. Setting thus $\sigma_{T}=\Sigma, \sigma_{L}=0$ in (22) we get an analytic expression for the hadron tensor $W_{\mu \nu}$ and are in a position to calculate the inelastic PS production cross section (21), summing over all hadron final states $|X\rangle$ with invariant mass $1.11 \leq M_{X} \leq 1.99 \mathrm{GeV}$.

As a specific check on our procedure we shall want to focus on a particular mass range, namely that of the $\Delta(1232)$ resonance. This can be achieved as follows. We multiply $\sigma_{\gamma \mathrm{p}}\left(s_{2}\right)$ in (21) by

$$
1=\int d M_{X}^{2} \delta\left(p_{X}^{2}-M_{X}^{2}\right)
$$

and define

$$
\begin{aligned}
\tilde{\sigma}_{\gamma \mathrm{p}}^{\gamma}\left(s_{2}\right):= & \int \frac{d^{3} k}{2 k^{0}(2 \pi)^{3}} \frac{1}{2 w\left(s_{2}, t_{1}=0, m_{\mathrm{p}}^{2}\right)} \frac{1}{2}\left(-g^{\mu \nu}\right) \\
& \left(4 \pi \alpha u_{P S}^{2} T^{2} \epsilon_{\mu \omega \alpha \beta} q_{1}^{\alpha} q_{2}^{\beta} \epsilon_{\nu \rho \gamma \delta} q_{1}^{\gamma} q_{2}^{\delta}\right) \frac{1}{t_{2}^{2}} \\
& (2 \pi)\left(2 m_{\mathrm{p}}\right) W^{\rho \omega}\left(p_{X}^{2}, t_{2}\right) m_{\mathrm{p}}^{2} \delta\left(p_{X}^{2}-m_{X}^{2}\right)
\end{aligned}
$$


Contracting the Lorentz indices in (25), inserting the explicit form of the hadron tensor and expressing the invariant functions through (22) we get finally for the cross section of inelastic PS production

$$
\begin{gathered}
\sigma^{\gamma}=\int_{M_{\min }}^{M_{\max }} d M_{X} \frac{2 M_{X}}{m^{2}} \int_{y_{\min }}^{y_{\max }} \frac{d y}{y} n(y) \int_{t_{2} \min }^{t_{2 \max }} d t_{2} \frac{d \tilde{\sigma}_{\gamma \mathrm{p}}^{\gamma}}{d t_{2}}\left(s_{2}\right), \\
\frac{d \tilde{\sigma}_{\gamma \mathrm{p}}^{\gamma}}{d t_{2}}=\frac{m^{2} T^{2} u^{2} \Sigma\left(M_{X}^{2}, t_{2}\right)}{64 \pi^{2}\left(s_{2}-m_{\mathrm{p}}^{2}\right)^{2} t_{2}^{2}} \frac{M_{X}^{2}-m_{\mathrm{p}}^{2}}{m_{\mathrm{p}}^{4}+\left(M_{X}^{2}-t_{2}\right)^{2}-2 m_{\mathrm{p}}^{2}\left(M_{X}^{2}+t_{2}\right)} \\
\left\{m_{P S}^{4}\left(m_{\mathrm{p}}^{2}-M_{X}^{2}\right)^{2}-2 t_{2} m_{P S}^{2}\left(M_{X}^{2}\left(m_{P S}^{2}+M_{X}^{2}-s_{2}\right)+m_{\mathrm{p}}^{2}\left(s_{2}-M_{X}^{2}\right)\right)+\right. \\
t_{2}^{2}\left(m_{\mathrm{p}}^{4}+m_{P S}^{4}+M_{X}^{4}+2 m_{P S}^{2}\left(2 M_{X}^{2}-s_{2}\right)+2 m_{\mathrm{p}}^{2}\left(m_{P S}^{2}-s_{2}\right)-\right. \\
\left.\left.2 M_{X}^{2} s_{2}+2 s_{2}^{2}\right)+2 t_{2}^{3}\left(s_{2}-m_{\mathrm{p}}^{2}-m_{P S}^{2}-M_{X}^{2}\right)+t_{2}^{4}\right\}
\end{gathered}
$$

The integration limits with respect to $t_{2}$ are just the kinematical limits of the two by two process $\gamma+\mathrm{p} \rightarrow \mathrm{PS}+\mathrm{X}$ as given, for example, in [35].

In the second row of Tab. I we list our results for the total cross section of inelastic PS electroproduction in the photoproduction region (2), calculated from (26). These values

\begin{tabular}{|l||c|c|}
\hline & $\sigma^{\gamma}(\mathrm{e} \mathrm{p} \rightarrow \mathrm{ePS})$ & $\sigma^{\gamma}(\mathrm{e} \mathrm{p} \rightarrow \mathrm{ePSp})$ \\
\hline$\pi^{0}$ & $2.0 \mathrm{pb}$ & $78.1 \mathrm{pb}$ \\
\hline$\eta$ & $1.9 \mathrm{pb}$ & $56.4 \mathrm{pb}$ \\
\hline$\eta^{\prime}$ & $3.1 \mathrm{pb}$ & $83.6 \mathrm{pb}$ \\
\hline$\eta_{c}$ & $0.3 \mathrm{pb}$ & $3.83 \mathrm{pb}$ \\
\hline
\end{tabular}

Table 1: Total cross section for for inelastic and elastic PS electroproduction by photon exchange.

are compared in the third row of Tab. 1 to the results of elastic PS production calculated in terms of the EPA in [22]. As we can read off from Tab. 11 the contributions from the inelastic PS production integrated cross sections are very small compared to the elastic ones. However in experimental analyses one typically has to make additional cuts on the data and, of course, this will change the ratio of inelastic to elastic contributions. We explore this first for the $t_{2}$-distribution.

To compare with the results of [22] it is convenient to plot the differential cross section with respect to the logarithm of $-t_{2}$ rather than $d \sigma^{\gamma} / d t_{2}$. Our results for $\pi^{0}$ production are shown in Fig. 4, together with the results of [22] for the elastic case. The cross sections for the other pseudoscalar mesons in essence scale as the coupling constants $u_{P S}^{2}$. It is immediately clear that the large difference in integrated cross section between the elastic and inelastic case is coming from the region of very small $\left|t_{2}\right|$. In the limit $t_{2} \rightarrow 0$ $d \sigma^{\gamma} / d t_{2}$ goes to a constant for the break-up calculation and simultaneously the available phase space region in $t_{2}$ becomes smaller and smaller. This results in the decrease of the 
logarithmic distributions in Fig. 4 for $\left|t_{2}\right| \leq 10^{-2}$. In the case of the elastic production $d \sigma^{\gamma} / d t_{2}$ increases as $1 /\left|t_{2}\right|$ as $t_{2} \rightarrow 0$. The corresponding logarithmic distribution then shows a plateau which is cut off by the upper phase space limit of $t_{2}$ [22. Another and

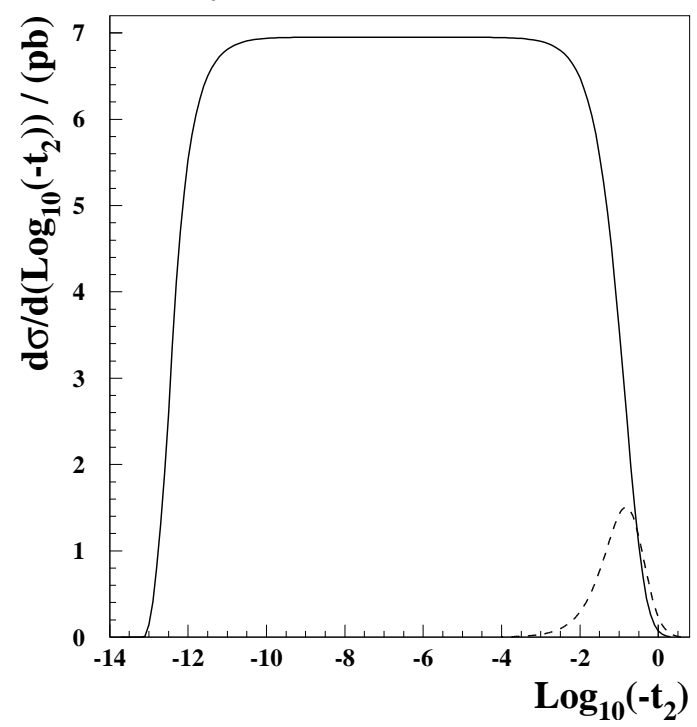

Figure 4: The $\log _{10}\left(-t_{2}\right)$ distributions for elastic $\pi^{0}$ production (solid line) taken from [22] compared to case of inelastic production (dashed line), integrated over the whole resonance region $\left(1.11 \leq M_{X} \leq 1.99 \mathrm{GeV}\right)$.

experimentally preferred variable to measure is $\left|\boldsymbol{k}_{T}\right| \equiv k_{T}$, the transverse momentum of the pseudoscalar mesons, defined relative to the beam axis. At HERA $k_{T}$ distributions can be measured for values of $k_{T}$ greater than $O(0.1 \mathrm{GeV})$. The photoproduction cuts of (2) restrict the transverse momentum of the incident photon to be smaller than $O(0.1$ $\mathrm{GeV}$ ), so in our case there is practically no distinction between the beam axis and the photon axis. Then within the accuracy of the EPA calculations we have $t_{2}=-k_{T}^{2}$. The complete electromagnetic result $d \sigma^{\gamma} / d k_{T}$ for all four pseudoscalars is shown in Fig. 5. We see that applying a cut $k_{T}>0.1 \mathrm{GeV}$ the break-up cross section represents a much larger fraction of the total then for the integrated cross sections (Table 1).

\subsection{The $\Delta(1232)$ resonance}

In this section we consider the hadron final state $|\mathrm{X}\rangle$ to be the resonance $\Delta(1232)$, and evaluate the cross section in two ways. Firstly we use the procedure of Section 3.1 but restrict ourselves to the $\Delta$ region which we define as $1.11 \leq M_{X} \leq 1.40 \mathrm{GeV}$ (see Fig. 3). Secondly the $\Delta$ is treated in the isobar model [36] as a stable particle (zero width approximation) of spin $3 / 2$.

A spin 3/2 particle can be described [37] by a vector-spinor $R^{\mu}$, which is contained in the direct product of the vector and the Dirac representation of the Lorenz group: 

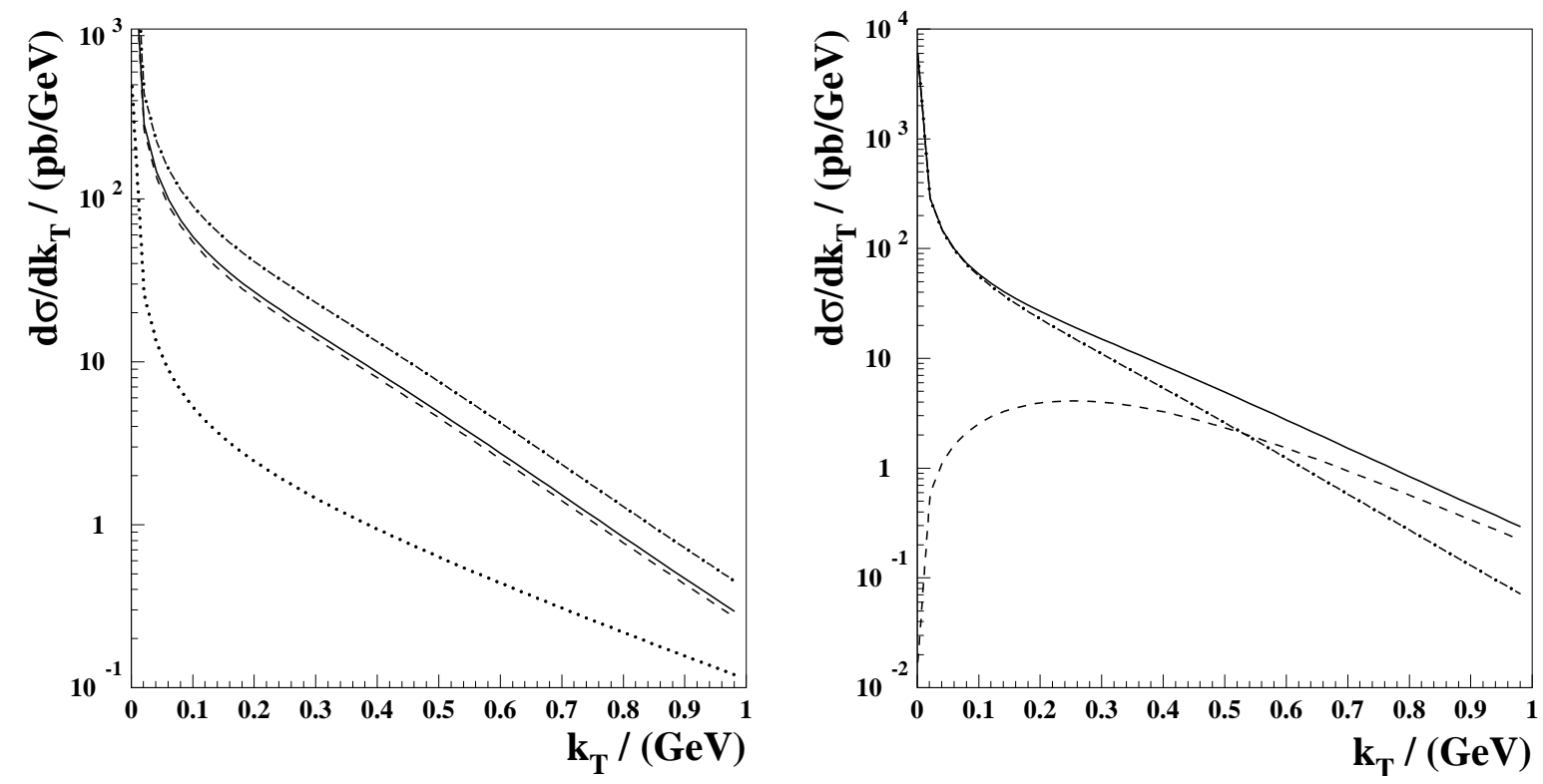

Figure 5: (a) The complete electromagnetic result for the $k_{T}$ distributions of $\pi^{0}$ (solid line), $\eta$ (dashed line), $\eta^{\prime}$ (dashed dotted line) and $\eta_{c}$ (dotted line) production. (b) The elastic (dashed dotted line) and the inelastic contribution (dashed line) together with the full electromagnetic result for pion production

$$
R^{\mu} \in\left(\frac{1}{2}, \frac{1}{2}\right) \otimes\left[\left(\frac{1}{2}, 0\right) \oplus\left(0, \frac{1}{2}\right)\right] .
$$

To project out the spin $3 / 2$ part of this reducible representation corresponding to the $\Delta$-particle one has to require $\gamma_{\mu} R^{\mu}=0$ and $p_{\Delta}^{\mu} R_{\mu}=0$ [37, 38] and that every component of $R^{\mu}$ fulfills the Dirac equation $\left(p_{\Delta} \cdot \gamma-m_{\Delta}\right) R_{\mu}=0$. Let $R_{\mu}\left(p_{\Delta}, i\right)(\mathrm{i}=1, \ldots, 4)$ be a set of normalised basis vector-spinors:

$$
\bar{R}_{\mu}\left(p_{\Delta}, i\right) R^{\mu}\left(p_{\Delta}, j\right)=\delta_{i j} \quad i, j=1, \ldots, 4 .
$$

To calculate the partial width or the total and differential cross sections we only need the polarisation sum $S_{\mu \nu}$. Using the above conditions we get for the polarisation sum $\left(v_{\Delta}=p_{\Delta} / M_{\Delta}\right)$

$$
\begin{aligned}
S^{\mu \nu}\left(p_{\Delta}\right) & =\sum_{i} R^{\mu}\left(p_{\Delta}, i\right) \bar{R}^{\nu}\left(p_{\Delta}, i\right) \\
& =\frac{1}{3}\left(\frac{\gamma \cdot v_{\Delta}+1}{2}\right)\left(3 g^{\mu \nu}-2 v_{\Delta}^{\mu} v_{\Delta}^{\nu}-\gamma^{\mu} \gamma^{\nu}\right)\left(\frac{\gamma \cdot v_{\Delta}+1}{2}\right) .
\end{aligned}
$$


The coupling of the $\Delta$ resonance to the proton and the photon is parametrised through

$$
\frac{2 e f_{\gamma R}}{M_{\Delta}} \epsilon_{\mu \nu \rho \sigma} p_{\Delta}^{\rho} p^{\sigma} G_{D}\left(q_{2}^{2}\right)
$$

which corresponds to the covariant formulation of the isobar model [36]. Here $G_{D}$ is defined in (23) and $f_{\gamma R}$ is the coupling constant. From (29) together with (28) we can calculate the $\Delta$ decay into a real photon and a proton. The result is

$$
\Gamma\left(\Delta^{+} \rightarrow \mathrm{p} \gamma\right)=\frac{e^{2} f_{\gamma R}^{2}}{3 \pi} \frac{\left(M_{\Delta}^{2}-m_{\mathrm{p}}^{2}\right)^{3}}{8 M_{\Delta}^{5}}
$$

This partial decay width is known experimentally [39, 40], $\Gamma\left(\Delta^{+} \rightarrow p \gamma\right)=0.65 \pm 0.02$ $\mathrm{MeV}$. Taking the central value from which it follows that $f_{\gamma R}=2.4$. Now everything is fixed and we can easily derive the hadron invariant functions $W_{1}^{\Delta}, W_{2}^{\Delta}$ :

$$
\begin{aligned}
W_{1}^{\Delta} & =\frac{f_{\gamma R}^{2} G_{D}^{2}}{3 M_{\Delta}^{4} m_{\mathrm{p}}^{2}}\left(\left(p q_{2}\right)^{2}-p^{2} q_{2}^{2}\right) f\left(p q_{2}, t_{2}\right) \delta\left(\left(p+q_{2}\right)^{2}-M_{\Delta}^{2}\right), \\
W_{2}^{\Delta} & =\frac{f_{\gamma R}^{2} G_{D}^{2}}{3 M_{\Delta}^{4} m_{\mathrm{p}}^{2}}\left(-p^{2} q_{2}^{2}\right) f\left(p q_{2}, t_{2}\right) \delta\left(\left(p+q_{2}\right)^{2}-M_{\Delta}^{2}\right) \\
f\left(p q_{2}, t_{2}\right) & =\left\{\left(m_{\mathrm{p}}+2 M_{\Delta}\right) m_{\mathrm{p}}^{2}+2\left(m_{\mathrm{p}}+M_{\Delta}\right) p q_{2}+m_{\mathrm{p}}\left(M_{\Delta}^{2}+t_{2}\right)\right\} .
\end{aligned}
$$

Having derived the hadron tensor it is then simply a task of contracting indices to get the cross section of photon proton scattering into a PS and the $\Delta^{+}$and, by applying the EPA, the total electroproduction cross section.

$$
\begin{gathered}
\sigma^{\gamma}=\int_{y_{\min }}^{y_{\max }} \frac{d y}{y} n(y) \int_{t_{2_{\min }}}^{t_{2 \max }} d t_{2} \frac{d \sigma_{\gamma \mathrm{p}}^{\gamma}}{d t_{2}}\left(s_{2}, t_{2}\right) \\
\frac{d \sigma_{\gamma \mathrm{p}}^{\gamma}}{d t_{2}}=\frac{e^{2} f_{\gamma R}^{2} G_{D}^{2} u_{P S}^{2} T^{2}}{384 m_{\mathrm{p}} M_{\Delta}^{3} \pi\left(s_{2}-m_{\mathrm{p}}^{2}\right)^{2} t_{2}^{2}}\left(\left(m_{\mathrm{p}}+M_{\Delta}\right)^{2}-t_{2}\right) \\
\left\{m_{P S}^{4}\left(m_{\mathrm{p}}^{2}-M_{\Delta}^{2}\right)^{2}-2 t_{2} m_{P S}^{2}\left(M_{\Delta}^{2}\left(m_{P S}^{2}+M_{\Delta}^{2}-s_{2}\right)+m_{\mathrm{p}}^{2}\left(s_{2}-M_{\Delta}^{2}\right)\right)+\right. \\
t_{2}^{2}\left(m_{\mathrm{p}}^{4}+m_{P S}^{4}+M_{\Delta}^{4}+2 m_{P S}^{2}\left(2 M_{\Delta}^{2}-s_{2}\right)+2 m_{\mathrm{p}}^{2}\left(m_{P S}^{2}-s_{2}\right)-\right. \\
\left.\left.2 M_{\Delta}^{2} s_{2}+2 s_{2}^{2}\right)+2 t_{2}^{3}\left(s_{2}-m_{\mathrm{p}}^{2}-m_{P S}^{2}-M_{\Delta}^{2}\right)+t_{2}^{4}\right\}
\end{gathered}
$$

This calculation is compared with the result for $d \sigma / d \log _{10}\left(-t_{2}\right)$ of Sect. 3.1, restricted to the $\Delta$ region in Fig. 6a. The comparison is quite satisfactory. The deviations can be understood qualitatively by plotting $\sigma_{T}=\Sigma$ from (23) at $M_{X}=1.215$ where the curve in Fig. 3 has its maximum, as a function of $t_{2}$ together with $\sigma_{T}$ calculated from $W_{1}^{\Delta}$ in (31) using the definition (22) and replacing the $\delta$-function in (31) with $1 /\left(\pi M_{\Delta} \Gamma\right)$ where $\Gamma$ is the total width of the $\Delta, \Gamma \approx 120 \mathrm{MeV}$ [35] (Fig. 6). For $\left|t_{2}\right| \leq 0.1 \mathrm{GeV}^{2}$ the fit (23) to the measured cross section $\Sigma$ is somewhat larger due to a significant S-wave component 

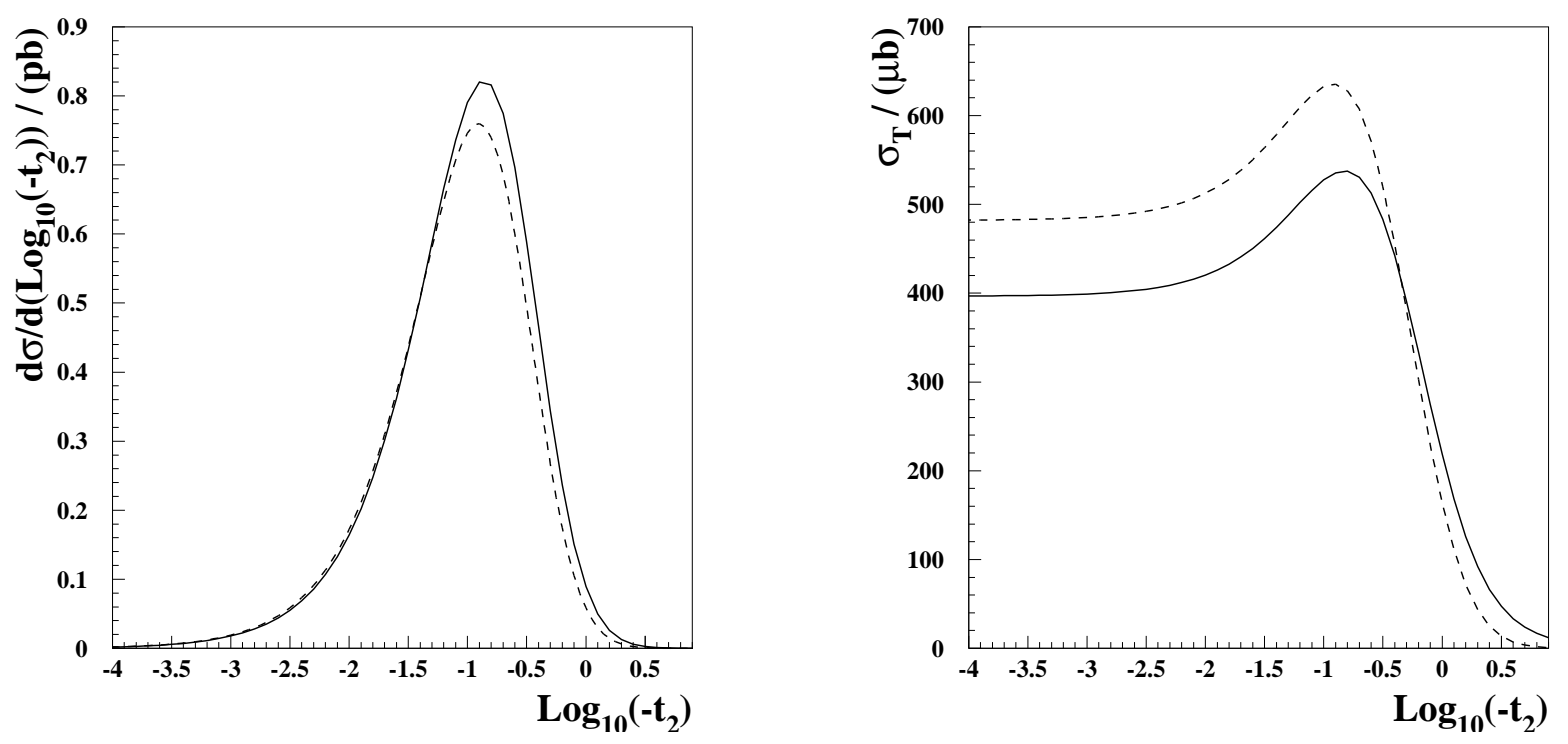

Figure 6: (a) $\log _{10}\left(-t_{2}\right)$ distributions for the delta resonance region, calculated from the $\gamma^{*}$ p cross section data as explained in Sect. 3.1, integrated over the invariant mass range $1.11 \leq M_{X} \leq 1.4 \mathrm{GeV}$ (dashed line), and from the isobar model (solid line). (b) $\sigma_{T}=\Sigma$ at $M_{X}=1.215 \mathrm{GeV}$ from (23) as a function of $t_{2}$ (dashed lines) compared with $\sigma_{T}$ calculated from $W_{1}^{\Delta}$.

which is absent in the calculation of the $\Delta$ alone but the shapes are nearly the same. For $\left|t_{2}\right| \geq 0.1 \mathrm{GeV}^{2}$ the model calculation of $\sigma_{T}$ decreases too slowly compared to experiment. In essence this translates directly into the shapes of Fig. 6a where for $\left|t_{2}\right| \leq 0.1 \mathrm{GeV}^{2}$ the differential cross section with respect to the logarithm of the exact result is a little bit larger than the one of the model calculation whereas for $\left|t_{2}\right| \geq 0.1 \mathrm{GeV}^{2}$ the model calculation overshoots the exact one.

\section{Conclusions}

We have calculated pseudoscalar electroproduction by odderon exchange with nucleon dissociation in a specific model which has proven successful in processes dominated by pomeron exchange. The principal conclusion is that the cross section is significantly larger for nucleon breakup than when the nucleon remains intact. We have also presented the results of an "exact" calculation of the same process by photon exchange. When combined with the previous calculation for the elastic case [22] we have a precise prediction for the purely electromagnetic cross section.

In Fig. 7 we show for $\pi^{0}$ production the distribution of the pion's $k_{T}$ summed over the elastic and inelastic channels for the electromagnetic and the odderon exchange. Interference terms are not taken into account here. Even taking the most pessimistic view of the uncertainties in the model the process should be observable at HERA. This would 


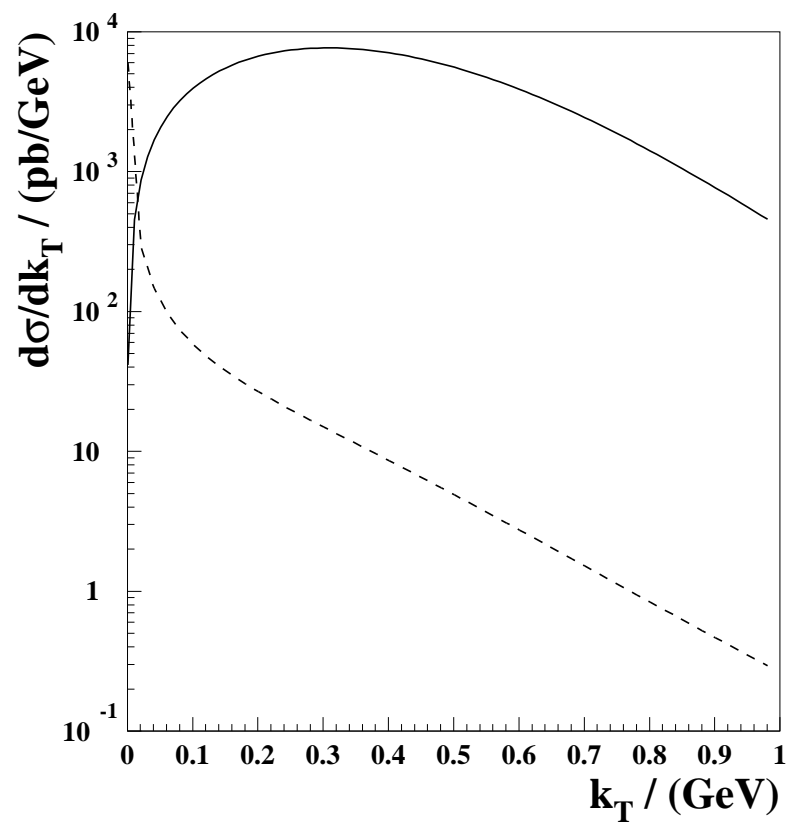

Figure 7: The $k_{T}$ distribution in pion production for odderon exchange (solid line) calculated from the amplitude (12) of Sect. 2 compared to the complete electromagnetic result (dashed line). Interference contributions are not taken into account.

establish the soft odderon as an exchange-object in high energy scattering on equal footing with the soft pomeron. On the other hand failure to observe any significant deviation from the electromagnetic result would be clear evidence for the complete failure of our model. One possible conclusion would then be that the soft odderon really does not exist and that our understanding of diffractive processes is much less than is believed.

The competing hadronic process, Reggeised $\omega$ exchange, can be estimated from what is known about $\pi^{0}$ photoproduction at low energies, $\sqrt{s} \leq 5.5 \mathrm{GeV}$ and the falloff with energy given by Regge theory: $d \sigma /\left.d t_{2}\right|_{\omega-\text { exch }} \sim s^{2 \alpha_{\omega}\left(t_{2}\right)-2}$ with $\alpha_{\omega}(0) \approx 0.18$ to 0.5 . The result is very much smaller than the calculated odderon contribution and is not a serious background to it. However it could conceivably be sufficiently large to interfere with the photon exchange amplitude and so simulate a very weakly-coupled odderon.

\section{Acknowledgements}

The authors would like to thank P. V. Landshoff, K. Meier, T. Berndt, S. Tapprogge, A. Likhoded, W. Buchmüller, E. Meggiolaro, M. Strikman, B. Nicolescu, A. Hebecker, H. J. Pirner, G. Kulzinger and S. Weinstock for useful discussions and correspondence. 


\section{References}

[1] A. Donnachie, P. V. Landshoff: Nucl. Phys. B 244, 322 (1984); Nucl. Phys. B 267, 690 (1986); Phys. Lett. B 185, 403 (1987), B 296, 227 (1992)

[2] L. Lukaszuk and B. Nicolescu: Nuov. Cim. Lett. 8, 405 (1973) ;

D. Joynson, E. Leader, C. Lopez, and B. Nicolescu: Nuov. Cim. A 30, 345 (1975);

B. Nicolescu, report hep-ph/9810465, 1998 and references therein

[3] C. Augier et al. (UA 4/2 Collab.): Phys. Lett. B 316, 448 (1993);

N. A. Amos et al., (E-710 Collab.): Phys. Rev. Lett. 68, 2433 (1992)

[4] L. N. Lipatov: Phys. Lett. B 251, 284 (1990); Nucl. Phys. Proc. Suppl. C 18, 6 (1990);

P. Gauron, L. N. Lipatov, and B. Nicolescu: Phys. Lett. B 304, 334 (1993); Z. Phys. C 63, 253 (1994).

[5] N. Armesto and M. A. Braun: Preprint DESY-97-150, hep-ph/9708296 (1997);

M. A. Braun: On the odderon intercept in the variational approach, hep-ph/9801352 (1998).

[6] B. V. Struminskii: Phys. Atom. Nucl. 57, 1398 (1994).

[7] G. P. Korchemsky: Nucl. Phys. B 462, 333 (1996);

R. A. Janik and J. Wosiek, Phys. Rev. Lett. 79, 2935 (1997); Solution of the odderon problem, hep-th/9802100 (1998); New results on the odderon in QCD, hep-th/9805135 (1998).

[8] R. Kirschner, private communication.

[9] P. V. Landshoff, O. Nachtmann: Z. Phys. C 35, 405 (1987)

[10] O. Nachtmann: Ann. Phys. 209, 436 (1991)

[11] H. G. Dosch, Phys. Lett. B 190, 177 (1987); H. G. Dosch, Yu. A. Simonov, Phys. Lett. B 205, 339 (1988); Yu. A. Simonov, Nucl. Phys. B 307, 512 (1988)

[12] A. Krämer, H. G. Dosch: Phys. Lett. B 252, 669 (1990), B 272, 114 (1991);

H. G. Dosch, E. Ferreira, A. Krämer: Phys. Lett. B 289, 153 (1992);

H. G. Dosch, E. Ferreira: Phys. Lett. B 318, 197 (1993)

[13] H. G. Dosch, E. Ferreira, A. Krämer, Phys. Rev. D 50, 1992 (1994)

[14] H. G. Dosch, T. Gousset, G. Kulzinger, H. J. Pirner: Phys. Rev. D 55, 2602 (1997);

M. Rueter, H. G. Dosch: Phys. Rev. D 57, 4097 (1998);

E. Ferreira, F. Pereira, Phys. Rev. D 55, 130 (1997), ibid. D 56, 179 (1997).

O. Nachtmann, in Perturbative and Nonperturbative Aspects of Quantum 
Field Theory, edited by H. Latal and W. Schweiger (Springer Verlag, Berlin, Heidelberg 1997)

[15] H. G. Dosch, T. Gousset, H. J. Pirner: Phys. Rev. D 57, 1666 (1998)

[16] G.Kulzinger, H.G.Dosch, H.J.Pirner, report hep-ph/9806352, 1998, to appear in Eur. Phys. J. C

[17] E. Berger, O. Nachtmann, report hep-ph/9811306, to appear in Eur. Phys. J. C

[18] M. Rueter, H. G. Dosch, Phys. Lett. B 380, 177 (1996)

[19] P. Gauron, B. Nicolescu, Phys. Lett. B 238, 406 (1990)

[20] A. Schäfer, L. Mankiewicz, and O. Nachtmann: Diffractive $\eta_{c}, \eta, \eta^{\prime}, J / \psi$ and $\psi^{\prime}$ production in electron-proton collisions at HERA energies in: Proceedings of the Workshop Physics at HERA, 1991 (W. Buchmüller and G. Ingelman, eds.), DESY, Hamburg (1992).

[21] J. Czyżewski, J. Kwieciński, L. Motyka, and M. Sazikowski: Phys. Lett. B 398, 400, (E) (1997); Erratum: ibid B 411, 402 (1997);

R. Engel, D. Y. Ivanov, R. Kirschner, and L. Szymanowski: Eur. Phys. J. C 4, 93 (1998).

[22] W. Kilian, O. Nachtmann, Eur. Phys. J. C 5, 317 (1998)

[23] M. Rueter , H.G. Dosch , O. Nachtmann, report hep-ph/9806342,

Phys. Rev. D 59, 014018 (1999)

[24] S. Tapprogge, Ph.D. Thesis, Report HD-IHEP 96-19, and private communication.

[25] V. M. Budnev, I. F. Ginzburg, G. V. Meledin, V. G. Serbo, Phys. Rep. 15, 181 (1975).

[26] T. Schäfer, E. V. Shuryak, J. J. M. Verbaarschot, Nucl. Phys. B412, 143 (1994)

[27] M. Rüter, Ph.D. thesis, University of Heidelberg, 1997.

[28] M. Wirbel, B. Stech, M. Bauer, Z. Phys. C29, 637 (1985)

[29] A. Donnachie, P. V. Landshoff, Phys. Lett. B348, 213 (1995)

[30] D. Aston et al., Nucl. Phys. B189, 15 (1981) and B209, 56 (1982)

[31] ZEUS collaboration, Eur. Phys. J. C2, 247 (1998); C. Adloff et al., Z. Phys. C75, 607 (1997)

[32] O. Nachtmann, Elementary Particle Physics, Concepts and Phenomena, Springer-Verlag Berlin/Heidelberg/New York (1990) 
[33] F. W. Brasse et al., Nucl. Phys. B 15, 413 (1976)

[34] L. N. Hand, Phys. Rev. 129, 1834 (1963)

[35] R. M. Barnett et al., (Particle Data Group), Eur. Phys. J. C 3, 1 (1998)

[36] J. S. Bell, S. M. Berman, Nuovo Cim. 25, 404 (1962); S. M. Berman, M. J. Veltman, Nuovo Cim. 38, 993 (1965);

J. S. Bell, in hadronic interactions of electrons and photons, edited by J. Cumming and H. Osborn (Academic Press, London and New York, 1971);

O. Nachtmann, in Weak Interactions, edited by M. K. Gaillard and M. Nicolic (Institut Nationale de Physique Nucleaire et de Physique Particule, Paris 1977)

[37] W. Rarita, J. Schwinger, Phys. Rev. 60, 61 (1941)

[38] S. Weinberg, The Quantum Theory of Fields , Cambridge University Press (1996)

[39] R. H. Dalitz, D. G. Sutherland, Phys. Rev. 146, 1180 (1966).

[40] F. A. Berends, D. L. Weaver, Nucl. Phys. B30, 575 (1971). 